\title{
Drainage apparatus for treating pneumothorax
}

\author{
Harry YJ Wu, MD, DPhil \\ Medical Ethics and Humanities Unit, Li Ka Shing Faculty of Medicine, The University of Hong Kong, Pokfulam, Hong Kong
}

https://doi.org/10.12809/hkmj-hkmms201910

This drainage apparatus (Fig), collected by the Hong Kong Museum of Medical Sciences and stored in a custom wooden case, is not an uncommon artefact. It does, however, tell a distinctive story of Hong Kong people's lungs. The apparatus was donated by Dr Chiu Kwong Yu in 1999 and consists of two 1-L glass bottles containing sterile solutions, each measuring $21 \mathrm{~cm}$ tall and $11.50 \mathrm{~cm}$ in diameter. The fluid in two bottles has deteriorated over time and become darkened. Attached to the case door is a glass barometer about $26 \mathrm{~cm}$ long. Under the glass bottles is a small drawer that used to contain consumables, such as syringes, needles, scalpels, and blades. The apparatus was used as a closed drainage system, mainly for the treatment of pneumothorax and other conditions that require chest drainage, such as pleural effusion and haemothorax. The portable design made it a practical device that could be operated at the bedside.
Pneumothorax is a common lung condition. When a pneumothorax occurs, the negative pressure in the pleural cavity is disrupted, resulting in respiratory distress. If the condition is mild, a small amount of fluid or air may be absorbed by the body after sufficient rest. However, in a more severe situation, a drainage system is required to prevent the lung from collapsing. Such treatment, employing a siphon effect, can be dated to the late 19th century. In one of the earliest documented cases in 1875, the German internist Gotthard Bülau described using drainage to treat an empyema as an alternative to the then-popular rib resection. He first used a trocar to puncture the pleural space and then introduced a rubber catheter with a distal clamp to create a closed water-seal system. The end of the catheter was immersed in a bottle one-third full of antiseptic solution and then unclamped, resulting in a siphon drainage apparatus and allowing pus to flow from

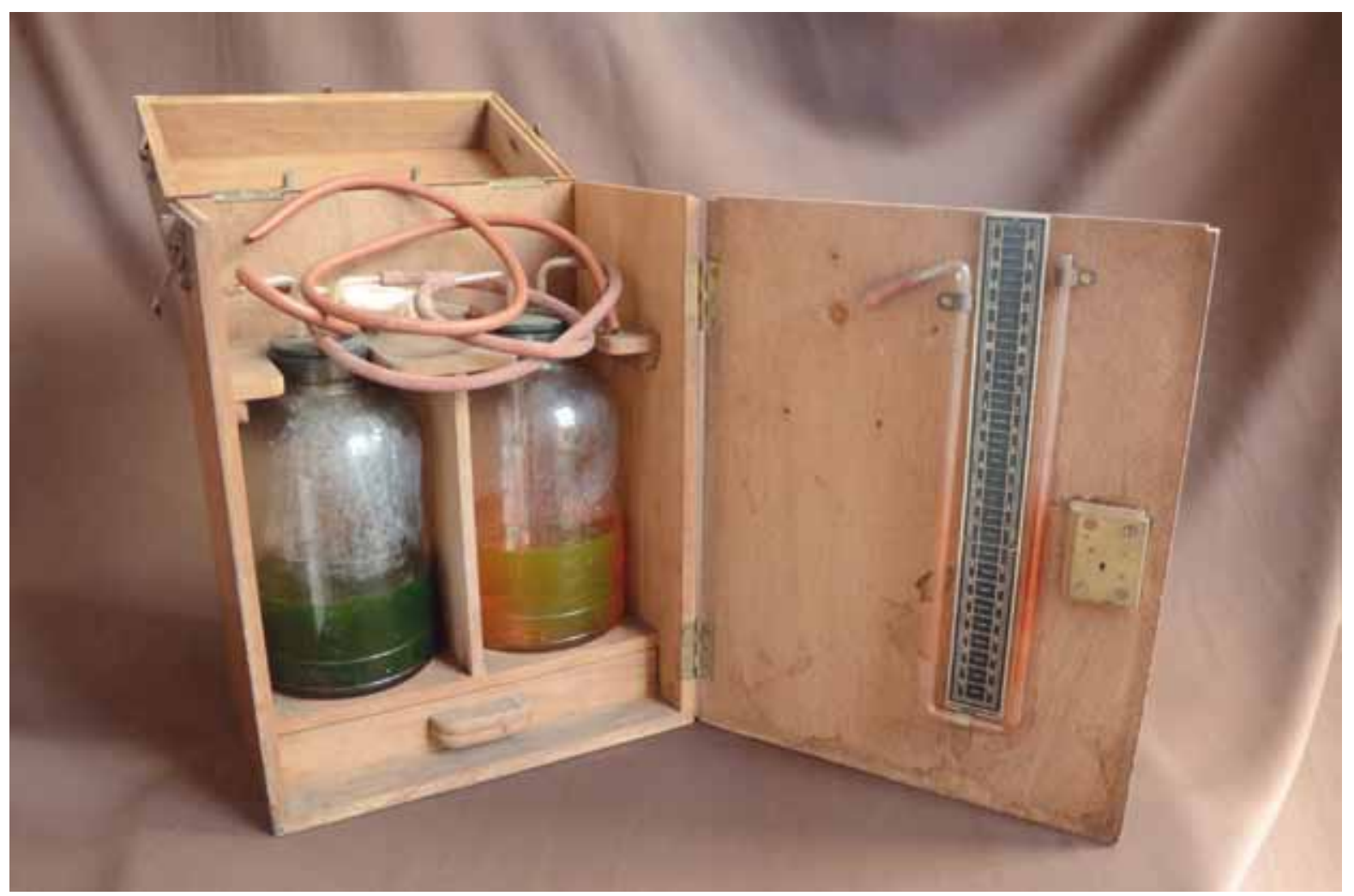

FIG. Drainage apparatus for the treatment of pneumothorax, stored in a custom wooden case (photo courtesy: Hong Kong Museum of Medical Sciences) 
the chest cavity. ${ }^{1}$

In modern times, several basic components are needed to drain the fluid or air out of a pleural cavity. This usually comprises a closed sterile and disposable system containing one or multiple chambers with a one-way valve that can prevent return of air or fluid into the patient. The main part of the apparatus collected by the museum was manufactured by Kelvin \& Hughes, a British company still in business that specialises in the design and manufacture of instruments related to navigation. However, the spindle-shaped glass compartment containing two Chinese characters shows clear signs that the entire apparatus might be a convenient assemblage of various sources. It was likely used most extensively in the 1960s; in 1963, closed thoracotomy drainage with a Foley catheter and two-bottle suction system was advocated as the preferred treatment for pneumothorax and significant haemothorax. ${ }^{2}$ While in use, the two bottles and spindle-form glass device containing sterilised cotton wool were connected by rubber tubes. One end of the apparatus connected the chest catheter that was inserted into the patient's pleura. The treatment could last from 1 day to 2 weeks, depending on how well the patient responded to the procedure. $\mathrm{X}$-ray plain films were sometimes useful for doctors to evaluate the remaining air or fluid in the pleura.

In the early days of Hong Kong's history, certain populations were more prone to developing pneumothorax. Resulting from poor sanitation and occupational hazards, these were mostly patients with more fragile lung conditions, such as those living with tuberculosis or pneumoconiosis. It is also worth noting that, from the 1930s to the 1950s, pneumothorax was employed as a treatment for tuberculosis. Before the advent of anti-tuberculosis drugs, there was no specific treatment for this infection, although surgical treatment could be offered in some early cases. In the 1930s, the three most commonly performed operations were sectioning of the phrenic nerve, creation of an artificial pneumothorax, and thoracoplasty by removal of some ribs. ${ }^{3}$ The Annual Medical Report of the government for 1934 reported that the University Medical Unit at Government Civil Hospital had started a special clinic for artificial pneumothorax of pulmonary tuberculosis cases. ${ }^{4}$ In 1938, artificial pneumothorax treatment was carried out in all suitable male cases at Queen Mary Hospital with 'gratifying results.' Such treatment gradually became less common in the 1950s, owing to the availability and effectiveness of anti-tuberculosis medication. However, it is understandable that pleural drainage might have continued to be used extensively, because of the high incidence of pneumothorax and lung cavity infection among the many tuberculosis patients at that time.

In modern times, treatment for pneumothorax is no longer complex. Most spontaneous pneumothorax patients will recover with sufficient bed rest and nutrition support. The most commonly used device for drainage is a plastic three-chamber unit. These include a collection chamber, a waterseal chamber, and a suction control chamber, which are interconnected. Despite international guidelines now advocating the use of simple aspiration and small-bore chest catheters, in Hong Kong, intercostal chest tube insertion remains popular. ${ }^{6}$ Therefore, this drainage apparatus represents, not an outdated practice, but conceivably only a change in materials.

\section{References}

1. Meyer JA. Gotthard Bülau and closed water-seal drainage for empyema, 1875-1891. Ann Thorac Surg 1989;48:597-9.

2. Felton WL 2nd. Initial evaluation and management of the patient with a chest injury. Am J Surg 1963;105:445-53.

3. Li SF. Indications, contraindications and end results in the surgical treatment of pulmonary TB. Caduceus 1931;10:167-77.

4. Wong TW. Plombage treatment for pulmonary tuberculosis in Hong Kong. Hong Kong Med J 2014;20:268.

5. Wilkinson PB. Report of the University Professorial Unit. Annual Medical Report for the Year Ending. $1938: 77$.

6. Chan JW, Ko FW, Ng CK, et al. Management of patients admitted with pneumothorax: a multi-centre study of the practice and outcomes in Hong Kong. Hong Kong Med J 2009;15:427-33. 